\title{
Saliency detection based on adaptive boundary
}

\author{
Chen Wang \\ 1. Northwestern Polytechnical University \\ 2. Air Force Engineering University \\ Xi'an 710072, China \\ e-mail:wwangchen77@163.com
}

\author{
Yangyu Fan \\ Northwestern Polytechnical University \\ Xi'an 710072, China
}

\author{
Lei Xiong \\ Air Force Engineering University \\ Xi'an 710038, China
}

\begin{abstract}
Background detection has been successful in solving many vision tasks. However, there is a challenging problem that they usually fail to face the situation that object and background are similar. In this paper, we present a novel saliency detection method to address this issue. Firstly, we estimate the rough background probability which can characterize the spatial layout of image regions. Secondly, we propose a robust background measure, called adaptive boundary. It is closer to salient object than boundary of image and can help to distinguish the foreground from background more efficiently. Then, we rank the similarity of the image regions using manifold ranking. The saliency value of each image region is defined based on its relevance to the adaptive boundary. Lastly, the saliency map is generated to exploit integrating saliency results of ranking based on adaptive boundary and boundary of image. Experimental results prove that the proposed algorithm outperforms many of the recent state-of-art and classical algorithms on several datasets.
\end{abstract}

Keywords - Background detection, Saliency detection, Adaptive boundary, Manifold ranking.

\section{INTRODUCTION}

Saliency detection is to identify the most important part of a scene. With the development of technology, this study has been got more attention in recent years. So more and more saliency methods have been proposed and have got many applications (e.g. image editing [1], image segmentation [2], object detection and recognition [3], and image cropping [4]). Previous saliency methods mostly belong to bottom-up methods and focus on some features of object and background, such as intensity, color and edge et al. The most widely utilized feature is the contrast between object and background.

Many contrast based methods $[5,6,7,8,9,10,11]$ are successful in saliency detection. But they are unsuccessful when background and background are dissimilar, or object and background are similar. This make the saliency difference between object and background is too small to get satisfying result, illustrated in Fig.1.In this example, there are two kinds of backgrounds which are very different. Hierarchical saliency (HS)[5] as a better saliency detection method based on contrast produces unsatisfying results(Fig.1(b)).

Some methods $[12,13,14]$ are built on boundary prior accord- ing to another assumption that image boundary regions are mostly background. Recently, a manifold ranking(MR) method that exploits the each of four image boundaries as the labelled queries is proposed[12]. Its main observation is that the background often presents local or global appearance connectivity of image boundary. The saliency value of each region is computed based on its relevance to boundary of image. This approach achieved satisfactory result, especially to complex background. However, it is fail when salient object is similar to boundary, illustrated in Fig.1. By working with a great deal of images, we observe that salient object is usually very different from its closely regions in human vision and the regions can be similar to image boundary or dissimilar. Instead of assuming the image boundary is background[12,13,14], the proposed method states that an image patch is background when the region is close to salient object and differ from salient object. In this work, we proposed a novel adaptive boundary to characterize the spatial layout of image background regions with respect to salient object. We exploit these cues to compute region saliency based on the improved ranking result of superpixels. In ranking, we exploit the regions closely with salient object as the queries and the saliency is computed based on graph structure.

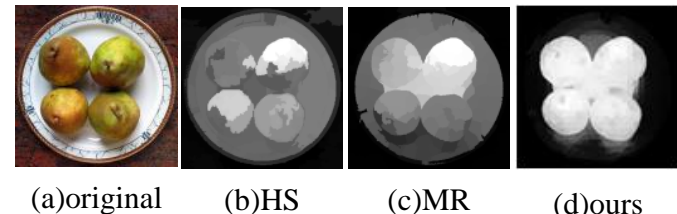

Fig.1 Saliency detection result of different methods

\section{ADAPTIVE BOUNDARY}

In [12], the proposed method exploit the each of four image boundaries as queries in the manifold ranking, the other nodes are ranked on their relevance to the queries.But in some images, the queries based on image boundary for saliency detection may be incorrect. Fig.1 (c),(d)show the saliency map using MR[12] and our proposed method respectively. In the example, the boundary of image is similar to salient object in color space, so it can not completely represent the characteristic of background. MR exploit the four boundaries of image as queries, it will get 
the low precision saliency map. In the result of MR, the salient object and a part of background are viewed as foreground. We need to find a method to ascertain the more fitting queries which can help to distinguish the salient object from background.

In [15], a concept called boundary connectivity is proposed. This index can characterize the spatial layout of image regions and the experiments have proven that it was robust background detection. In this paper, we also exploit this method to ascertain the approximately range of salient object in one image, illustrated in Fig. 2. In the example, we use the each of four rectangle boundaries(Fig.2(d)), which include salient object, as queries in ranking instead of image boundary in MR(Fig.2(b)). We call this novel boundary is adaptive boundary. See from Fig.2(b),(d), the adaptive boundary is the closer to salient object than image boundary. Based on these new queries, the saliency algorithm can be easy to distinguish salient object from background. In this work, the saliency of each node is defined as its ranking score.

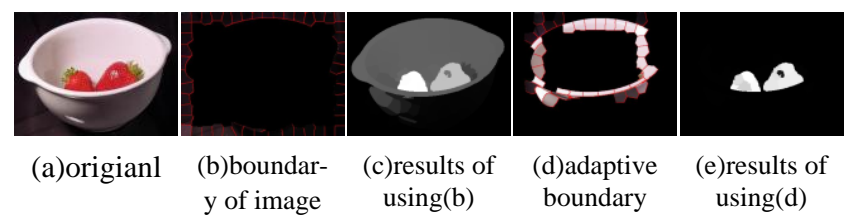

Fig.2 Saliency maps using different boundaries.

\section{IMPROVED GRAPH-BASED RANKING}

Ranking method can exploit the intrinsic manifold structure of data for graph labelling. In saliency detection, we want to design a novel ranking function to define the relevance between unlabelled nodes and queries. We firstly segment the source image to many regular regions using the algorithm of SLIC [16]. For all the experiments in this paper, we set the 600 pixels in a superpixel.Then viewing the segmented image as a set of points: $X=\left\{\mathrm{x}_{1}, \mathrm{x}_{2}, \cdots, \mathrm{x}_{n}\right\} \in \mathbf{R}^{h \times w}, \mathrm{~h}$ and $\mathrm{w}$ are the height and the width of image. Each point is a superpixel. Let $\mathrm{f}: X \rightarrow \mathbf{R}$ is the ranking function, its value is saliency value of the each superpixel. Vector $y=\left[\mathrm{y}_{1}, \mathrm{y}_{2}, \cdots, \mathrm{y}_{n}\right]^{T}$ is a indicate vector, in which $y_{i}=1$ if $x_{i}$ is a query, and $y_{i}=0$ otherwise. Then we define a graph $G=(V, E)$ based on the set of points, where $\mathrm{V}$ are the nodes based on $\mathrm{X}, \mathrm{E}$ are the edges. The affinity matrix $W_{i j}=\exp \left[-d_{c o l}^{2}\left(x_{i}, x_{j}\right) / 2 \sigma^{2}\right]$ defined by the distance in the CIE LAB space between two $\operatorname{nodes}\left(x_{i}, x_{j}\right)$. Similar to the manifold ranking [17,12], we proposed a novel manifold ranking function via manifold ranking.

The ranking function in [17] is:

$$
\mathrm{f}^{*}=(I-\alpha S)^{-1} y
$$

where $\mathrm{I}$ is an identity matrix, $\alpha$ is parameter in $[0,1)$. $S=D^{-1 / 2} W D^{-1 / 2}$ is a normalize matrix, in which $\mathrm{D}$ is the diagonal matrix with $(i, i)$ equal to the sum of the $i-t h$ row of $\mathrm{W}$.

The manifold ranking can be viewed as a classification prob- lem. Above ranking function in saliency detection has been changed for another format[12]:

$$
\mathrm{f}^{*}=(\mathrm{D}-\alpha \mathrm{W})^{-1} \mathrm{y}
$$

In eq.(2), the ranking function only considerate the distance in color space, the geometry distance and salient object position has not got full consideration. For example, if two paris of nodes in one image have equal color distance, the ranking function will get the equal value no matter their position according to eq.(2). In this work, we take color and geometry distance into consideration, and propose follow ranking function:

$$
\mathrm{f}^{*}=\left[\frac{\mathrm{D}-\alpha \mathrm{W}+\mathrm{A}}{\mathrm{W} * \mathrm{~A}}\right]^{-1} \mathrm{y}
$$

where matrix $\mathrm{A}$ is diagonal weighted matrix based on geometry distance:

$$
\mathrm{A}=\operatorname{diag}\left(\sum_{\mathrm{j}} \mathrm{d}_{\text {geo }}\left(\mathrm{x}_{\mathrm{i}}, \mathrm{x}_{\mathrm{j}}\right) * \operatorname{bdCon}\left(\mathrm{x}_{\mathrm{j}}\right)\right)
$$

where vector bdCon is the boundary connectivity[15] and it can give the robust background detection. $d_{g e o}\left(x_{i}, x_{j}\right)$ is the geometry distance of nodes. Matrix A represent the influence of the position in ranking function.

The graph structure as shown in Fig.3(a), the nodes V denote superpixels. It is the boundary map from segmenting the original image(Fig.3(b)) by SLIC algorithm. The green line alongs the four sides of image boundary indicate the background queries of MR (Fig.3(c)). The red line alongs the four sides of adaptive boundary indicate the background queries of our method(Fig.3(e)). The thickened line indicates the range of salient object. On the graph, we get two close-loop. The inner loop(red line) constraint can improve the precision of MR, and the outer loop(green line) constraint add global improvement of the proposed method.

\section{INTEGRATION AND IMPORVEMENT}

\section{- A. Integration}

Exploiting the each of four adaptive boundaries as queries, we will get four saliency maps. Then we need to integrate them for the initial saliency map. Similar to integrating method in [12], we also integrate the four saliency maps by the following process:

$$
\mathrm{S}_{\mathrm{bga}}(\mathrm{i})=\mathrm{S}_{\mathrm{ta}}(\mathrm{i}) \times \mathrm{S}_{\mathrm{ba}}(\mathrm{i}) \times \mathrm{S}_{\mathrm{la}}(\mathrm{i}) \times \mathrm{S}_{\mathrm{ra}}(\mathrm{i}) \quad \mathrm{i}=1,2, \cdots, \mathrm{N}
$$

where $\mathrm{S}_{\mathrm{bga}}(\mathrm{i})$ denotes saliency value of $i$-th node(i.e. $i$-th superpixel) on graph. The four variables on right side of the eq.(5) are the four saliency maps using the top, bottom, left and right of adaptive boundaries as queries respectively.

Adaptive boundary usually is the more nearer to the salient object than boundary of image. The proposed method can provide two benefits. Firstly, it provides the efficient and precise queries. This make the saliency algorithm can be more sensitive to salient object, especially as salient object is similar to background, or some complex background. Secondly, the adaptive 


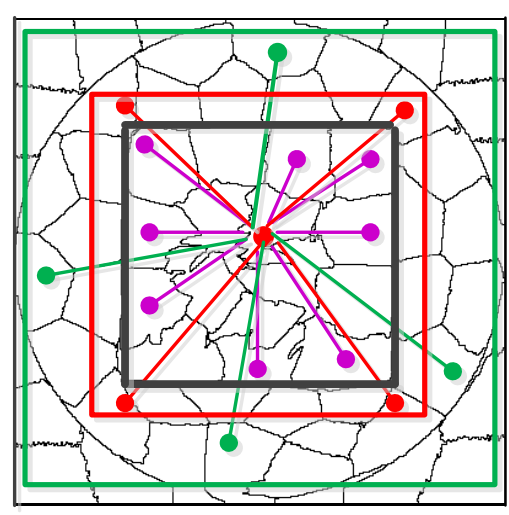

(a)Our graph structure

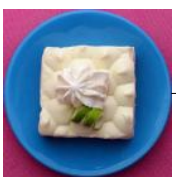

(b)original

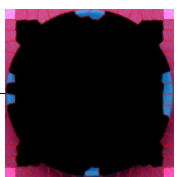

(c)queries 1

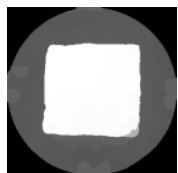

(d)ranking using queries 1

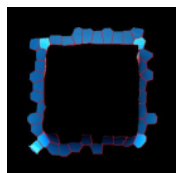

(e)queries 2

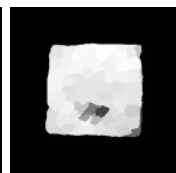

(f)ranking using queries 2

Fig. 3 Our graph structure and saliency maps

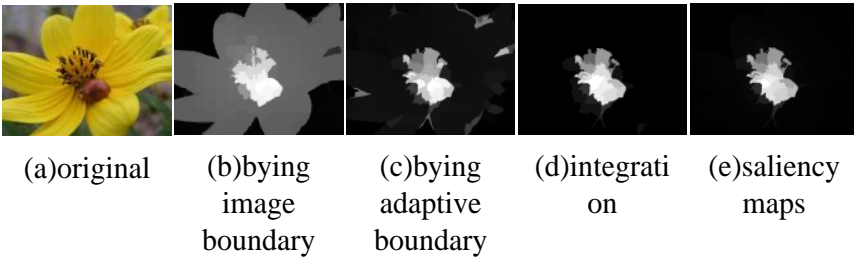

Fig. 4 The example of itegration

boundary based on robust background detection is robust in saliency detection. As shown in Fig.4(c), the saliency maps generated only using adaptive boundary. Due to the fitting queries, the superpixels with the salient object have high saliency value.

\section{B. Improving the saliency map}

However, see from Fig.4(c), a part of the background nodes are incorrect in saliency map. The reason is the adaptive boundary is close to salient object, and it may be similar to image boundary or dissimilar. When dissimilar, the proposed method may make some nodes on nearby image boundary are highlighted. So we need to use the saliency map based on image boundary to improve.

$$
\left.\mathrm{S}(\mathrm{i})=\left(1-\overline{\mathrm{f}}^{*}(\mathrm{i})\right) *\left(\mathrm{~S}_{\text {bgb }}(\mathrm{i}) * \mathrm{~S}_{\text {bga }}(\mathrm{i})\right)+\lambda \mathrm{S}_{\text {bgb }}(\mathrm{i})\right)
$$

where $S_{\text {bgb }}$ is the saliency map based on image boundary, $\mathrm{S}_{\mathrm{bga}}$ is the saliency map based on adaptive boundary. S(i) denote the saliency value of $i$-th superpixel. $\overline{\mathrm{f}}^{*}$ denotes the normalized vector. $1-\overline{\mathrm{f}}^{*}$ (i) denote the ranking function of foreground which can measure the saliency of the nodes based on foreground as queries. $S_{\text {bgb }}$ (i) $* S_{\text {bga }}$ (i) denote the superpixel which is highlighted by the two different kinds of queries at the same time. $\lambda$ is adjustment parameter. We use the $S_{\text {bgb }}$ as the adjustment term which can improve the saliency value which is ignored by $\mathrm{S}_{\mathrm{bga}}$. At last, we exploit the ranking function to get final saliency map in eq.(6).
In the above final saliency map, some background nodes may not be adequately suppressed, see from Fig.4(d). In order to improve the saliency map, we use binary method in[12] to deal with this problem and get the ideal final saliency maps.

\section{EXPERIMENTS}

My experiments are doing based on three datasets: ASD[10] is widely used in most all saliency methods. This dataset has 1000 images and provide the human labeled masks for salient objects. The other two datasets are MSRAhard[11] and DUT-OMRON dataset and each of them has more than 5000 images.

We compare our method with recent state-of-art methods and classical methods, including region contrast(RC)[18], saliency filter(SF) [19], geodesic saliency(GS)[13], hierarchical saliency(HS) [5],robust background detection(RBD)[15] and manifold ranking(MR)[12].

In this work, we use standard precision-recall(PR) rate, Fmeasure and mean absolute error(MAE)[15] to evaluate the saliency result. The PR curves can compare the saliency map against the ground truth by producing binary maps at different thresholds in $[0,255]$. The F-measure is the computed using the weighted of precision and recall:

$$
F_{\beta}=\frac{\left(1+\beta^{2}\right) \text { Precision }+ \text { Re } \text { call }}{\beta^{2} \text { Precision }+ \text { Re } \text { call }}
$$

MAE is the average per-pixel difference between the binary ground truth and the saliency map. This index can measure how close a saliency map is to the ground truth. Fig.5-7 show the evaluation results using above index on three datasets: the PR rate, F-measure and MAE of the proposed method is better than other methods. Fig. 8 shows the saliency results of previous saliency methods: the proposed algorithm outperforms many of the recent state-of-art and classical algorithms on several datasets.

\section{CONCLUSION}

In this work, we proposed a saliency method based on adaptive boundary. We adopt an improved ranking function 
which considerate geometry distance and background distribution between superpixels to get the more ideal saliency map than previous methods. We evaluate the proposed method on three datasets and testify the significant performance. Our future work will focus on how to improve the method to adapt to multiple dispersive salient objects.

\section{ACKNOWLEDGEMENTS}

This work is supported by The National Science Foundation of China(No.61379104).
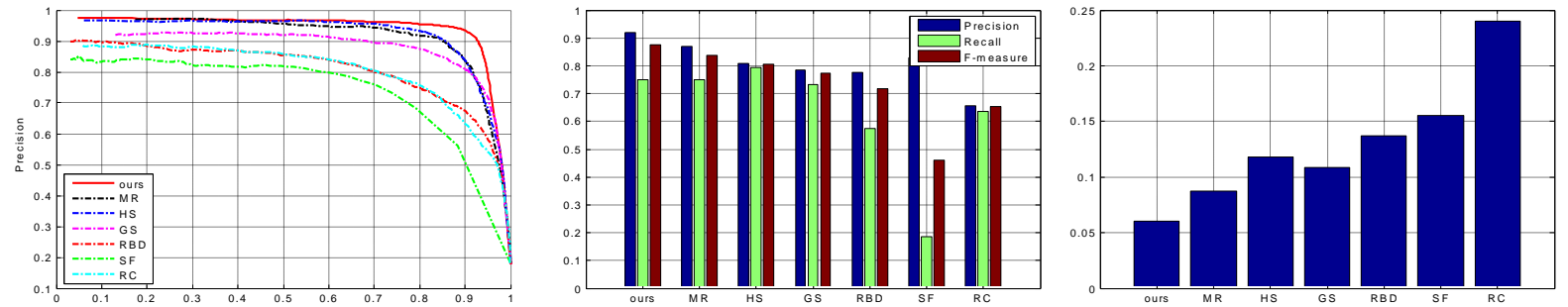

Fig.5 PR curves(left)、F-measure(center) and MAE(right) of different methods on ASD dataset.
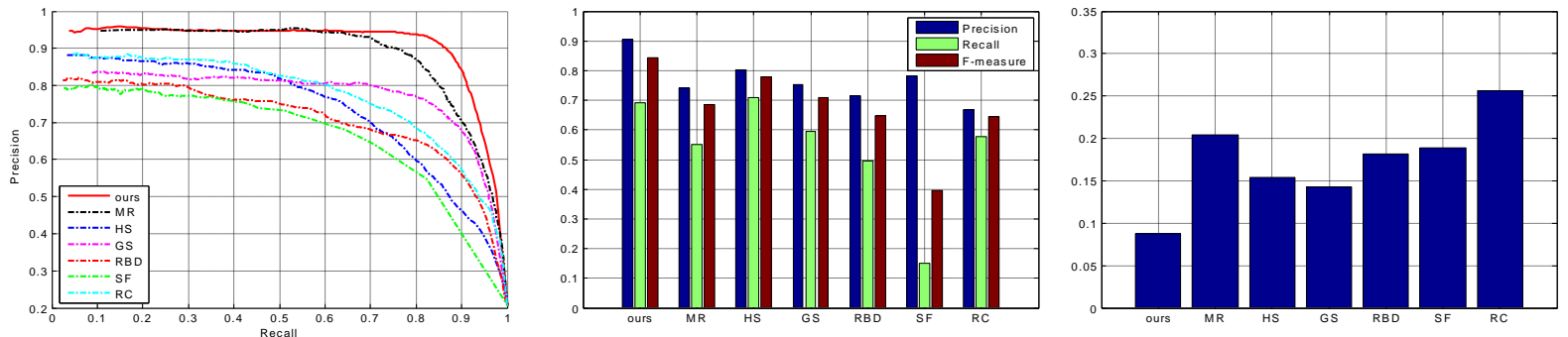

Fig.6 PR curves(left)、F-measure(center) and MAE(right) of different methods on MSRA-hard dataset.
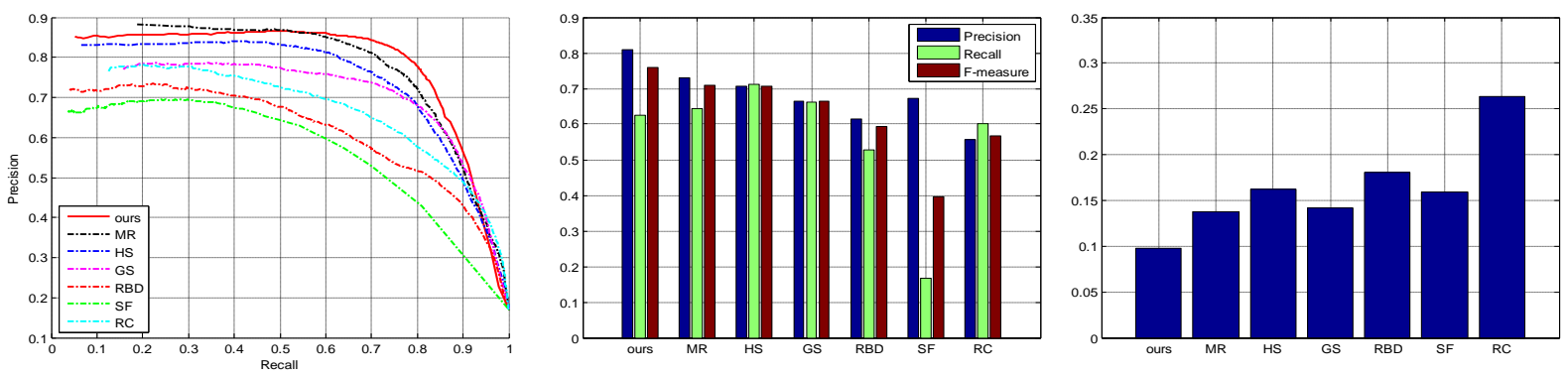

Fig.7 PR curves(left)、F-measure(center) and MAE(right) of different methods on DUT-OMRON dataset.
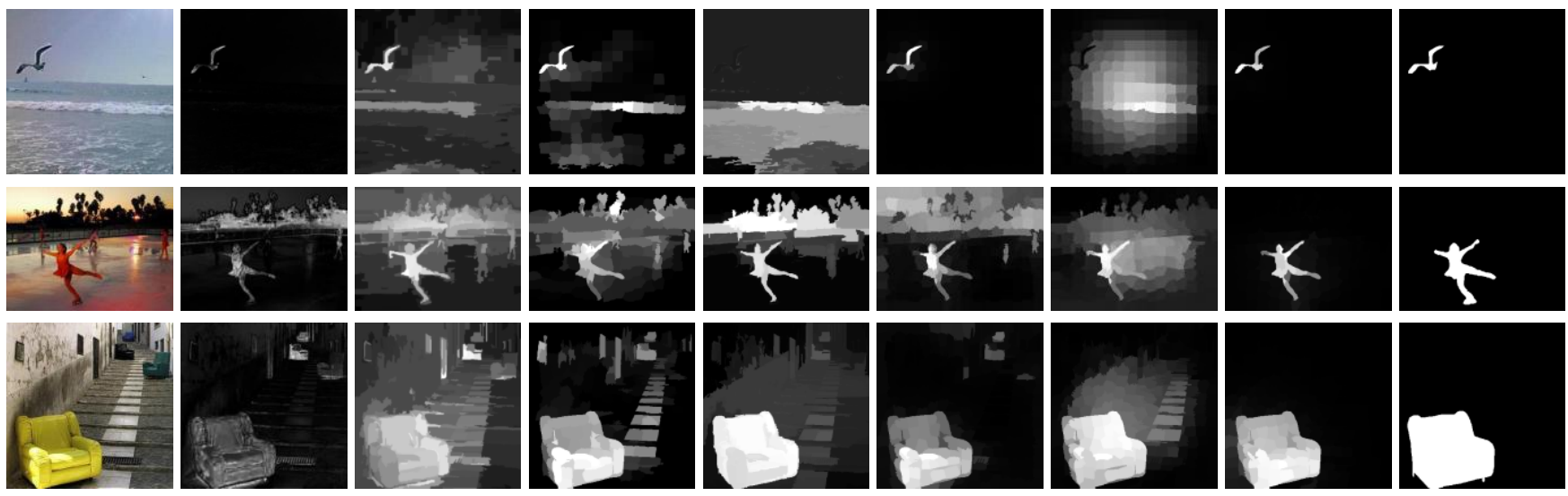


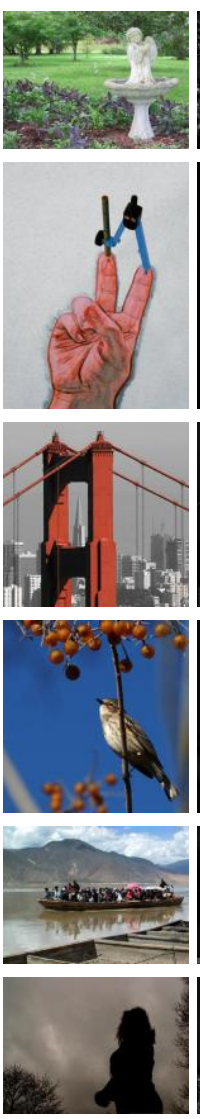

(a) original
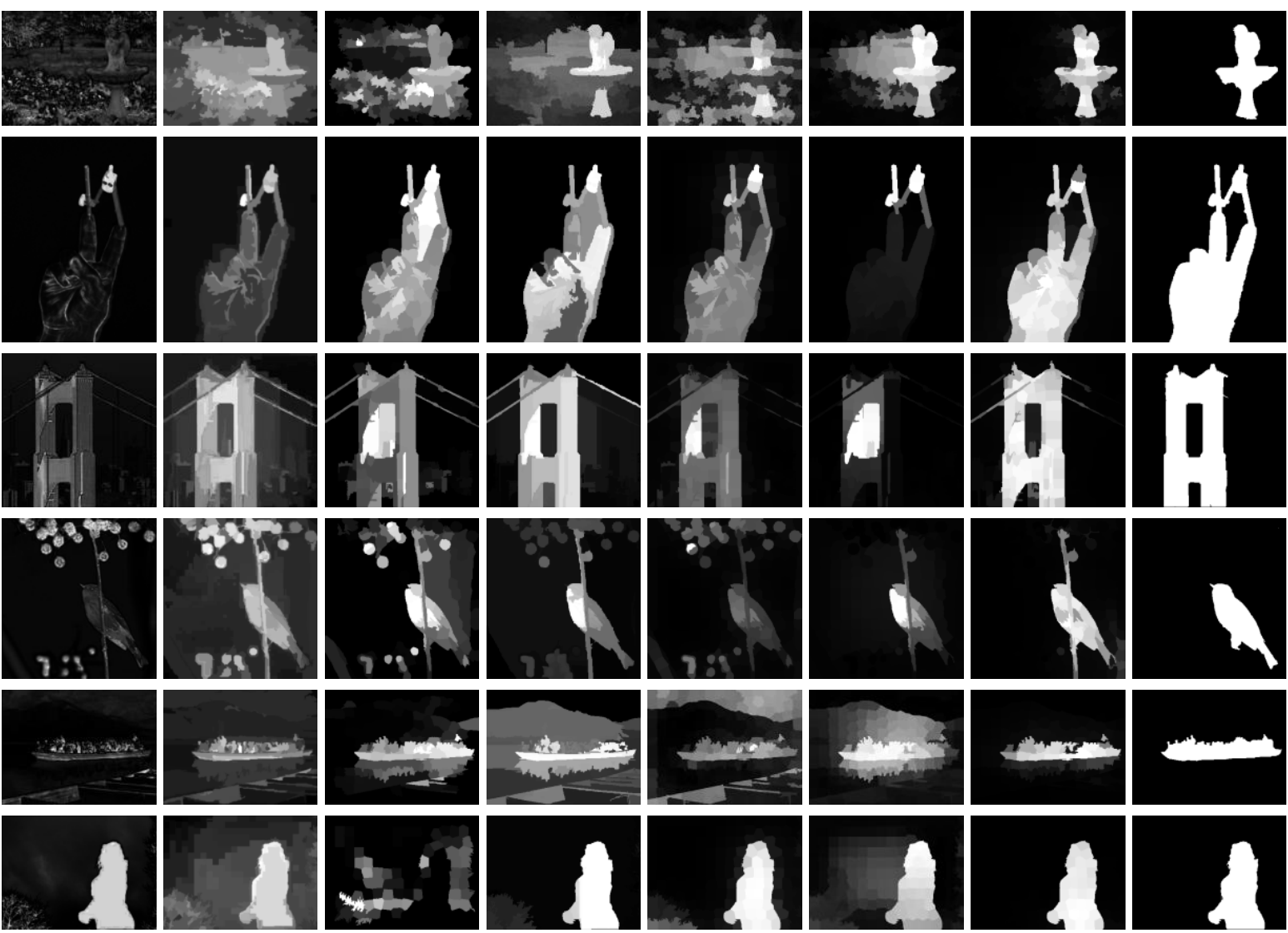

(e)HS

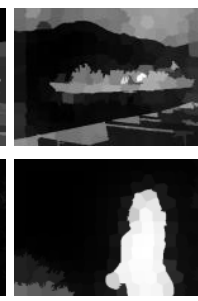

(f)RBD

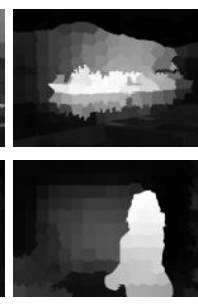

(g)MR
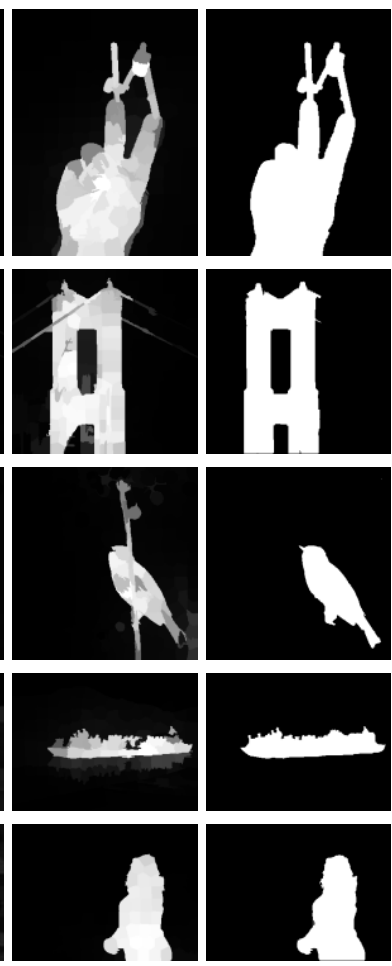

(h)ours

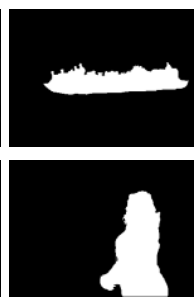

(i)GT

Fig.8 saliency results of different methods on DUT-OMRON (1-2 rows),MSRA(3-4 rows),ASD(5-6 rows)

\section{REFERENCES}

[1] S. Goferman, L. Zelnik-Manor, and A. Tal. "Context-aware saliency detection.” IEEE Trans., PAMI, 34(10), pp.1915-1926, 2012.

[2] B. C. Ko and J.-Y. Nam. "Object-of-interest image segmentation based on human attention and semantic region clustering." IEEE Trans. , ICPR, Hong Kong,vol.ICPR_4, pp:45-48, 2006.

[3] U. Rutishauser, D. Walther, C. Koch, and P. Perona. "Is bottom-up attention useful for object recognition." IEEE Trans. , CVPR, Pasadenavo, USA, vol. CVPR_2,pp:37-44, 2004.

[4] L.Marchesootti, C.Cifarelli, and G.Csurka. "A Framework for visual saliency detection with applications to image thumbnailing." IEEE Trans., ICCV, Kyoto, Japan, pp:2232-2239, 2009.

[5] Q. Yan, L. Xu, J. Shi, and J. Jia. "Hierarchical saliency detection.” IEEE Trans., CVPR, Portland, pp:1155-1162, 2013.

[6] L.Wang, J.Xue, N.Zheng, and G.Hua. "Automatic salient object extraction with contextual cue." IEEE Trans, ICCV, Barcelona, pp:105-112, 2011.

[7] Z. Jiang and L. S. Davis. "Submodular salient region detection.” IEEE Trans, CVPR, Portland, pp:2043-2050, 2013.

[8] D.Klein and S.Frintrop. "Center-surround divergence of feature statistics for salient object detection." IEEE Trans., ICCV, Barcelona, pp:2214-2219, 2011.
[9] M.-M. Cheng, J. Warrell, W.-Y. Lin, S. Zheng, V. Vineet, and N. Crook. "Efficient salient region detection with soft image abstraction." IEEE Trans., ICCV, Sydney, VIC, pp:1529-1536, 2013. [10] R.Achanta, S.Hemami, F.Estrada, and S.Susstrunk. "Frequency-tuned salient region detection." IEEE Trans., CVPR, Miami, FL, pp:1597-1604, 2009.

[11] T.Liu, J.Sun, N.Zheng, X.Tang, and H.Shum. "Learning to detect a salient object." IEEE Trans., Minneapolis, MN, pp:1-8, 2007.

[12] C. Yang, L. Zhang, H. Lu, X. Ruan, and M.-H. Yang. "Saliency detection via graph-based manifold ranking." IEEE Trans., CVPR, Portland, OR, pp:3166-3173, 2013.

[13] Y. Wei, F. Wen, W. Zhu, and J. Sun. "Geodesic saliency using background priors." Springer Berlin Heidelberg, ECCV, Florence, Italy, pp 29-42, 2012.

[14] H. Jiang, J. Wang, Z. Yuan, Y. Wu, N. Zheng, and S. Li. "Salient object detection: A discriminative regional feature integration approach.” IEEE Trans., CVPR, Portland, OR, pp:20832090, 2013.

[15] Wangjiang Zhu, Shuang Liang and Yichen Wei, Jian Sun. "Saliency optimization from robust background detection." IEEE Trans., CVPR, Columbus, OH, pp:2814-2821, 2014.

[16] R.Achanta, A.Shaji, K.Smith, A.Lucchi, P,Fua, and S.Susstrunk. "Slic superpixels compared to state-of the-art super- 
pixel methods." IEEE Transactions on Pattern Analysis and Machine Intelligence, vol.34, pp: 2274-2281, 2012.

[17] D.Zhou, J.Weston, A. Gretton, O.Bousquet, and B. ScholKopf. "Ranking on datat manifolds." Advances in Neural Information Processing Systems, MIT Press, USA,vol:16, pp:1-7, 2004.

[18] M.M. Cheng, G. X. Zhang, N. J. Mitra, X. Huang, and S.M. Hu. "Global contrast based salient region detection." IEEE Trans., CVPR, Providence, RI,pp: 409 - 416 , 2011.

[19] F.Perazzi, P.Krahenbuhl, Y. Pritch, and A. Hornung. "Saliency filters:Contrast based filtering for salient region detection." IEEE Trans., Providence, RI ,pp: 733 - 740 , 2012. 